\title{
Studying Various Software Testing Trends for Computers and Mobile Application
}

\author{
Rahul Sharma \\ Secretary, \\ Auricle Global Society of Education and Research, India \\ rahulmnu1@gmail.com
}

Abstract: - This paper will explain the latest testing trends. Now-a-days the software testing process is not just related to finding errors and looking for latest technologies but also to improve the testing techniques and finding new ways of testing which can help to predict future threats etc. This paper will throw light on trends which are used by most of the organisations these days.

Keywords: - Testing Trends, Software testing, Artificial intelligence, Machine Testing.

Introduction: - Software testing process has been growing ever since it has been introduced in the market. This process helps the organisations to know the efficiency of their products and services as it gives the opportunities to the testers to know errors in the product before giving it to the end users. Since the organisations believes that it is very important to test their services on the basis of security, usability, efficiency etc much before it is given to the end users. So, there are always ways and methods to improve the process based on previous experience of the software testing. In this modern time of latest technologies, there is not only scope of improvement in the testing methods but also the organisations are looking for such testing processes which will help to identify the future threats, errors, failure issues much in advance stage. Following are the latest software testing trends used by the organisations these days: -

1. Internet of things testing

2. Artificial intelligence

3. Machine Learning

4. Automation testing without using code

5. Security Testing

6. Agile automation testing

7. DevOps

8. Importance of Performance testing

9. Data Science

10. Having own testing centres

Internet of Things Testing: -This is the most-latest technology which is in demand these days. The meaning of this term is to use devices which are connected to each other via network connection and can be operated using one device. Few companies will have their own IoT but some companies hire the service from service providers. Hence, these devices are in demand and in coming future it will definitely in crease due to its many advantages. Therefor, it is very important for the testers to have testing tools so that the testing can be done easily. In some IoT, the providers will also provide testing tools but in some cases the testers need to buy the tools for testing process or also in some scenarios the open tools are also available for testing. For conducting IoT testing, it is very important to have testers who are highly qualified and have hands on experience on testing devices which are used in IoT If the testers do not have proper knowledge of IoT tools then they will not be able to test the devices present in this technology. In all the cases the IoT testers will make sure that they perform the following types of testing: - 


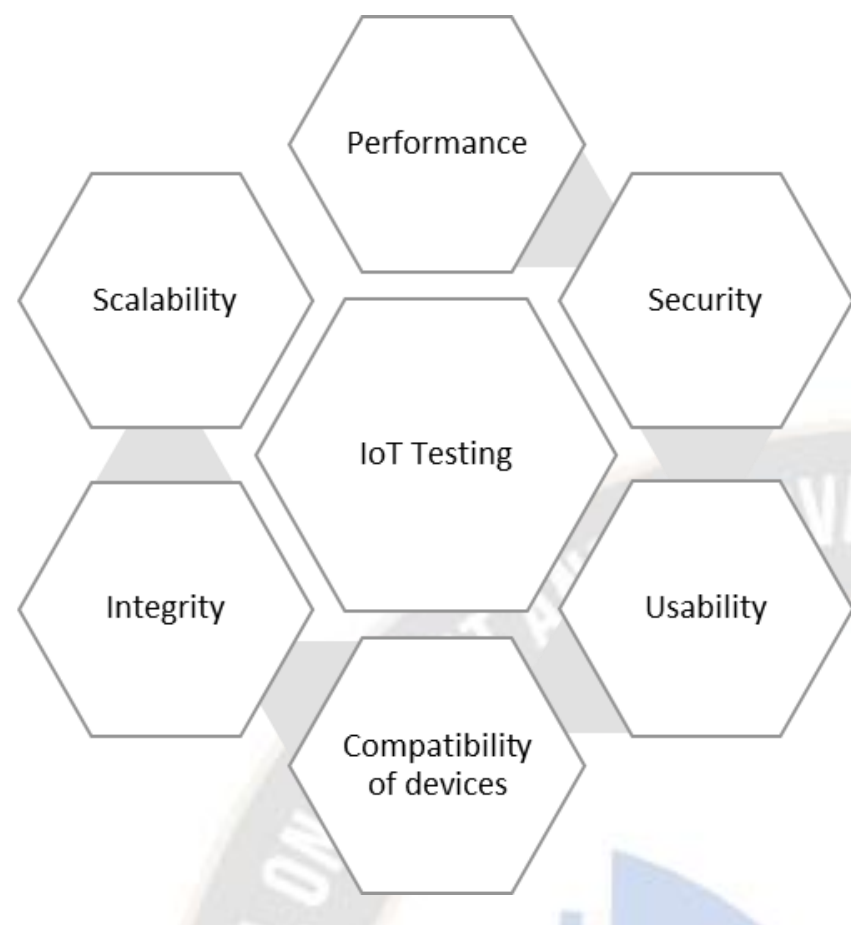

Figure 1 IoT testing

2. and 3. Artificial Intelligence and Machine Learning: - The use of artificial intelligence as well as machine learning have a great impact on the software testing of the services. They have not only improved the process of testing but has also helped to speed up the process of software testing. It has proved to be a boon in the field of software testing. It has been used for the following purposes: -
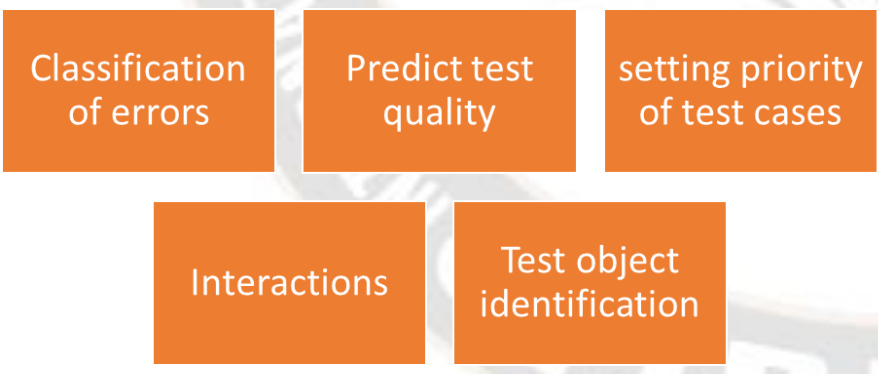

- Less time consuming: -This reduces the overall testing time process as it itself will do the testing based upon the programmed algorithms.

- Availability: - There is no such issue related to availability of the testers. AI is available for use $24 * 7$ hence it improves the process of testing.

- Repetitive tasks: - There are certain test procedures which needs repetition of the steps used to perform testing. It is kind of boring tasks after doing which the testers usually become bored. Hence, the AI can be programmed in such a way that it itself will repeat certain tasks which are important for the testing process.

- Quick Decision: - AI technology uses fast way to taking decisions unlike human beings. Testers being human takes a lot of time to make a perfect decision but AI technology helps to take fast decision and also conduct the testing based on that decision and give faster results.

Disadvantages of AI: -

- One of the disadvantages of AI testing is that as it needs less manpower, it will increase the job losing trends.

- AS everything in $\mathrm{AI}$ is programmed it will result in decrease in the efficiency of testers with which they work.

- It will also reduce the scope of improvement of knowledge of testers as everything is done by AI.

- Less innovations: The AI will keep repeating whatever is programmed in it which will reduce the chances of some new innovations. If the testers are working than there are chances that they find some new way of testing but this is not the case with AI.

- High Cost: - It is very difficult as well as costly to maintain the machinery used for AI. Hence, the cost factor is high.

Figure 2 Use of AI in software testing

Advantages of Artificial Intelligence in Software testing: - [1]

- Human Error can be minimised: -Human tends to make many mistakes. If we use AI then it helps to reduce the error rate as it is programmed and used. The catch here is that it should be programmed perfectly. 


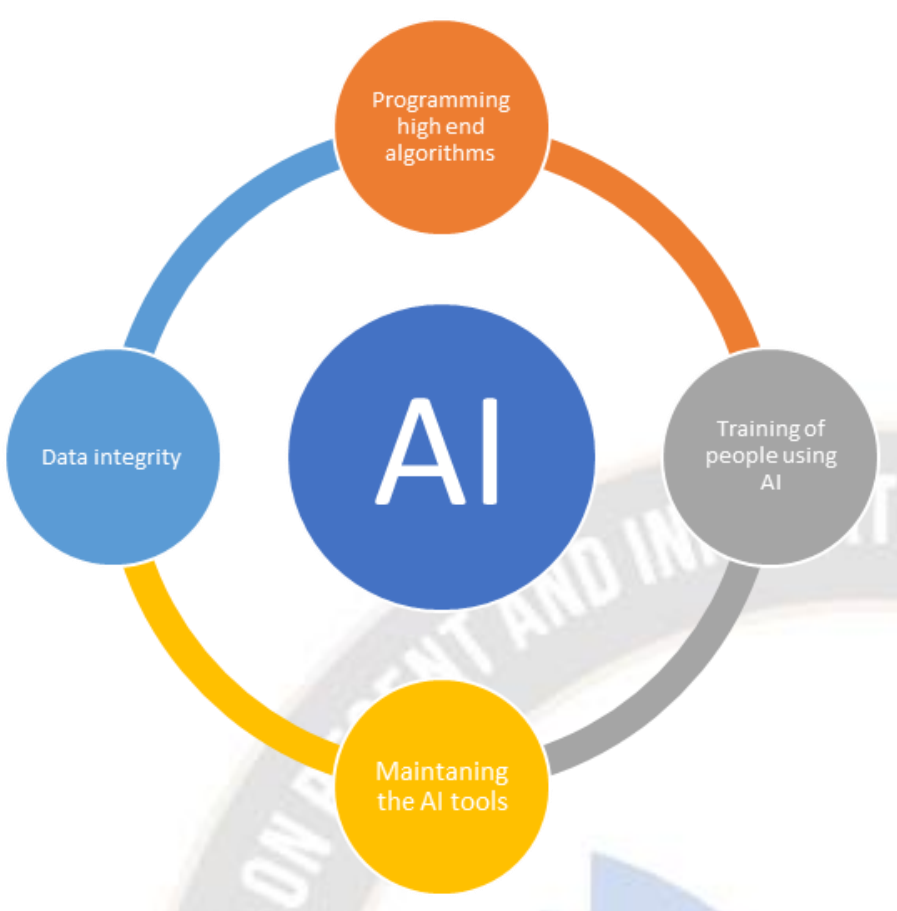

Figure 3 Key Challenges of AI Testing

4. Automation Testing without using code: -Automation testing tools haver changed the way of testing in many ways and has many advantages as it saves a lot of time and energy. There are many automation testing's tools which have reduced the time consumed while doing manual testing. So, many companies have adopted the method of automation testing. But now these days there are trends where automation testing is being done where there is no coding while writing of test scripts. There are many tools which provides codeless automation testing techniques. So, in future this method will be in trend where testers are using tools which gives them the opportunity of doing automation testing without writing scripts. Some of the codeless automation testing tools available are: - [2]

*UseTrace

*Leapwork

$*$ Ranorex

*CloudQA

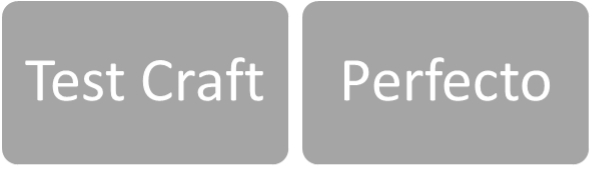

Sikuli

\section{Katalon Studio}

\section{Applitools}

Figure 4 Tools used for Codeless automation Testing

5.Security Testing: - Security testing is also very important type of testing which cannot be left unseen. As, there are latest technologies like internet of things and cloud computing, the security of the data being shared online is a basic need of the hour. There are many devices which are connected over network so it is the responsibility of the testers to make sure that there are no security threats to the data of the customers. They should test whether the devices are providing proper authorisation, authentication and also there is no leakage of the private information of the customers. In future as the demand for IoT, AI, Cloud computing will increase so will the security testing. The testers will pay more and more attention for the security testing.

6. Agile Automation Testing: - As automation testing helps the testers to save time it is in trend. Agile automation testing is the process of automation testing which gives more efficient results with the help of decreased life cycle. This is going to trend in future as testers are looking for more effective ways of performing automation testing. This has many benefits. [3]

Following is kept in mind while performing agile automation testing: - 


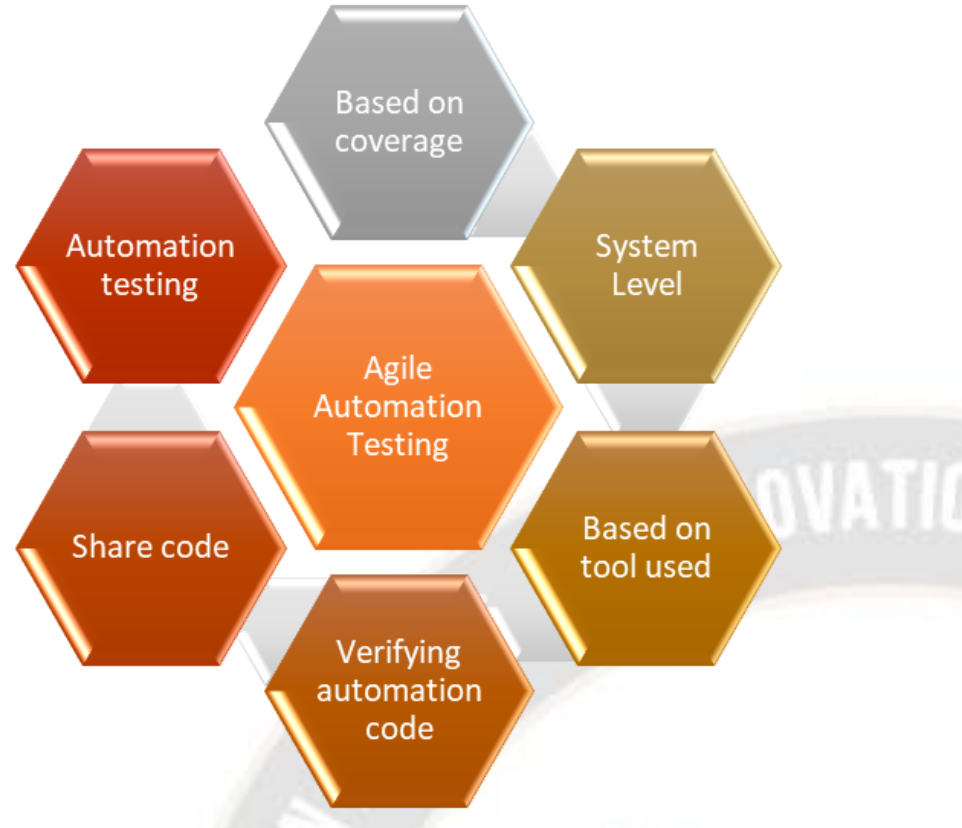

Figure 5 Agile Automation Testing

7. DevOps: - This is one of the tools that is used to collaborate between the developing team and the testing team. This tool makes sure that the quality of the product is good. These days both agile and DevOps is used to ensure the quality of the product. This will result in faster deployment of the products. Along with performing the testing of the product the role of the testers is to provide complete information about the quality of the product which is a continuous process.

8. Importance of Performance Testing: - This is also one of the most important part of the testing process as it will help to the testers to determine how the product or system under test will behave under different work load conditions. The point to be tested is that the product should function properly even under extreme work load conditions. This will also trend in future as it is very important type of testing which should be done before the product is made available to use for the end users.

9. Data Science: - The process of having centralised data which gives the option to analyse the data and interpret it in one place is called data science. The testers these days are not only looking for finding the error but also finding ways which will help to identify the threats which might occur in future. So, with the help of data centres this can be done. This is also in trend and will also increase in future as there are lot of IoT devices.

10. Having own Testing Centres: - The organisations these days are trying to have their own separate testing centres which will deal only with the testing of all the products and services provided by them to the end users. This will help them to increase the efficiency with which they work and helps them to improve their working capabilities which will result in business success. There are many advantages of having own centralised testing centres as it will help to save time and also it will give more appropriate testing results.

Conclusion: - This paper has discussed about various testing trends in todays time as well as the future if the software testing. The software testing is not just related to finding bugs and fixing them but also finding new ways to improve the quality of the products by determining the future failure chances in advance and try to avoid them before it is being used by the end user.

\section{References: -}

1.https://www.edureka.co/blog/what-are-the-advantages-anddisadvantages-of-artificial-intelligence/

2.https://dzone.com/articles/2020-and-top-codeless-testingtools-youd-better-kn

3.https://www.cigniti.com/blog/7-core-practices-of-agile-testautomation-

2/\#: :text=Agile\%20testing\%20helps\%20make\%20test,assur ance $\% 20$ and $\% 20$ quickens $\% 20$ application $\% 20$ delivery.\&text $=$ Implementation $\% 20 \mathrm{of} \% 20$ such $\% 20$ testing $\% 20$ therefore $\% 2$ 0requires $\% 20$ persistent $\% 20$ collaboration $\% 20$ between $\% 20$ the $\% 20$ different\%20teams. 